\title{
Effectiveness of Lower Energy Density Extracorporeal Shock Wave Therapy in the Early Stage of Avascular Necrosis of the Femoral Head
}

\author{
Yong Han, MD, June-Kyung Lee, MD, Bong-Yeon Lee, MD, \\ Hoi-Sung Kee, MD, Kwang-Ik Jung, MD, Seo-Ra Yoon, MD
}

Department of Physical Medicine and Rehabilitation, Gwangju Veterans Hospital, Gwangju, Korea

\begin{abstract}
Objective To evaluate the effectiveness of lower energy flux density (EFD) extracorporeal shock wave therapy (ESWT) in the early stage of avascular necrosis (AVN) of the femoral head.

Methods Nineteen patients and 30 hips were enrolled. All subjects received 4 weekly sessions of ESWT, at different energy levels; group A ( $n=15 ; 1,000$ shocks/session, EFD per shock $\left.0.12 \mathrm{~mJ} / \mathrm{mm}^{2}\right)$ and group $B(n=15 ; 1,000$ shocks/ session, EFD per shock $0.32 \mathrm{~mJ} / \mathrm{mm}^{2}$ ). We measured pain by using the visual analog scale (VAS), and disability by using the Harris hip score, Hip dysfunction and Osteoarthritis Outcome Score (HOOS), and the Western Ontario and McMaster University Osteoarthritis Index (WOMAC). To determine the effect of the lower EFD ESWT, we assessed the VAS, Harris hip score, HOOS, WOMAC of the subjects before and at 1, 3, and 6 months. Results In both groups, the VAS, Harris hip score, HOOS, and WOMAC scores improved over time ( $<<0.05)$. Conclusion Lower EFD ESWT may be an effective method to improve the function and to relieve pain in the early stage of AVN.
\end{abstract}

Keywords Femoral head necrosis, High-energy shock waves, Disability evaluation, Treatment outcome

\section{INTRODUCTION}

Avascular necrosis (AVN), also known as ischemic bone necrosis, osteonecrosis, bone infarction, aseptic necrosis, is cellular damage within the bone caused by interruption of the blood supply. It is reported that AVN occurs more frequently in the hip joint than in any other joint [1]. Since it was first reported as a "Coronary artery disease of the hip" in 1948, various approaches have been used to identify the cause and the natural history of the disease; however, yet the mechanisms remain uncertain with many etiological factors, and the disease is one of the

Received January 28, 2016; Accepted March 28, 2016

Corresponding author: Hoi-Sung Kee

Department of Physical Medicine and Rehabilitation, Gwangju Veterans Hospital, 99 Cheomdanwolbong-ro, Gwangsan-gu, Gwangju 62284, Korea. Tel: +82-62-602-6114, Fax: +82-62-602-6990, E-mail: no-gold@hanmail.net

ORCID: Yong Han (http://orcid.org/0000-0003-0147-1915); June-Kyung Lee (http://orcid.org/0000-0002-9900-0961); Bong-Yeon Lee (http://orcid. org/0000-0002-2975-6333); Hoi-Sung Kee (http://orcid.org/0000-0002-7683-8366); Kwang-Ik Jung (http://orcid.org/0000-0002-9754-5925); Seo-Ra Yoon (http://orcid.org/0000-0002-3694-0710).

@ This is an open-access article distributed under the terms of the Creative Commons Attribution Non-Commercial License (http://creativecommons.org/ licenses/by-nc/4.0) which permits unrestricted noncommercial use, distribution, and reproduction in any medium, provided the original work is properly cited. Copyright $\odot 2016$ by Korean Academy of Rehabilitation Medicine 
most common intractable diseases [2]. If it is not treated with adequate treatments, AVN of the femoral head progresses to femoral head depression and hip joint destruction, which require total hip replacement in the end.

Currently, the incidence of AVN of the femoral head is gradually rising along with the increased usage of steroids in numerous conditions. At the same time, the technological advances in radiologic techniques such as magnetic resonance imaging (MRI) and computed tomography have enabled an early diagnosis of the disease. With such trends, an early, effective treatment for AVN of the femoral head is drawings more attention.

Clinical treatment of AVN of the femoral head may be divided into two categories in general: surgical and nonsurgical treatments. While planning surgical treatment, potential risks, complications, and uncertain prognosis must be considered. Among surgical treatments, core depression with or without bone grafting is considered the gold standard femoral head preserving procedure. However, the prognosis after the procedure showed wide variations and the patients were often unsatisfied with the operation results, according to the previously reported cases and papers [3].

While treating AVN of the femoral head, the fundamental goal of the physician is to preserve the joint and its function. Thus, most physicians prefer non-surgical treatments to preserve the hip joint as much as possible, since AVN usually affects people between 30 and 50 years of age.

Non-surgical treatments, such as extracorporeal shock wave therapy (ESWT), hyperbaric oxygen therapy, and oral alendronate have been reported to be effective methods to treat AVN of the femoral head [4]. However, these treatments do not cure the disease; they are conservative methods of controlling pain and preserving the function. Therefore, various innovative attempts have been made for treating AVN of the femoral head with non-surgical treatment.

In order to set a standard procedure of ESWT for clinical application, the International Society for Medical Shockwave Treatment (ISMST) recommends a protocol, using $0.62 \mathrm{~mJ} / \mathrm{mm}^{2}$ of energy flux density (EFD) and 4,000 shocks delivered to the skin close to the damaged bone for AVN treatment. Other than the ISMST recommendation, there are numerous experimental and clinical approaches to preserve the joint and prevent progression to the secondary degenerative joint disease during the early stage of AVN of the femoral head [3-5]. Recently, several studies verified the effectiveness of the ESWT session under general anesthesia [3-5]. However, the ESWT protocol as recommended by the ISMST is difficult to execute at the clinical level; the low output of the energy that can be generated by the typical clinical machines poses a technical limitation. In an attempt to overcome this limitation, several studies assessed the effectiveness of low-EFD and their results suggest that the treatment at a low-EFD of 0.4 $\mathrm{mJ} / \mathrm{mm}^{2}$ is effective in alleviating the symptom [6].

The aim of this report is to evaluate the effectiveness of lower energy density ESWT, which is a more practical and realistic treatment option at the clinical level, than the previously used methods. The evaluations were made with respect to the degree of pain, radiological studies, and the improvement of, AVN of the femoral head at the fracture level.

\section{MATERIALS AND METHODS}

\section{Subjects}

Our patient pool comprised those who visited Gwangju Veterans Hospital from May 2013 to May 2015, with either unilateral or bilateral pelvic pain, gait disturbance, and radiologically diagnosed AVN of the femoral head using magnetic resonance imaging. Our inclusion criteria for the subjects were (1) age>30 years; (2) diagnosed as having grades from 1 to 3 of the Association Research Circulation Osseous (ARCO) classification of AVN. The exclusion criteria were (1) patients with comorbidities such as a neurologic disorder or a hemodynamically unstable system; (2) patients who have received steroid injection in the past 6 months; (3) patients with a contraindication found on MRI; (4) patients with a history of pelvic trauma. We recruited a total of 32 patients and 48 hips, and 19 patients and 30 hips met our inclusion criteria. Written informed consent was obtained from all subjects prior to the study. The study was performed in accordance with the principles set forth in the Declaration of Helsinki.

\section{Methods}

There are a number of clinical staging standards to determine the appropriate treatment of AVN of the femoral head; among these classifications, the ARCO classification is the most widely used method [5]. Therefore, we adopted the ARCO classification in our study. 


\section{ESWT}

We used the Dornier Epos Ultra (Dornier MedTech, Kennesaw, GA, USA), which was approved in 2001. Our ESWT has an electromagnetic generation source and its type is the defocused type; the machine's maximum level of EFD per shock was 20 gauge, which is equivalent to 0.32 $\mathrm{mJ} / \mathrm{mm}^{2}$, and the middle level of EFD per shock was 10 gauge, equivalent to $0.12 \mathrm{~mJ} / \mathrm{mm}^{2}$. We performed block randomization via a computerized program; an independent researcher assigned the subjects to either group A ( $\mathrm{n}=15 ; 1,000$ shocks/session, EFD per shock $0.12 \mathrm{~mJ} /$ $\mathrm{mm}^{2}$, the middle output) or group B ( $\mathrm{n}=15 ; 1,000$ shocks/ session, EFD per shock $0.32 \mathrm{~mJ} / \mathrm{mm}^{2}$, the maximum output), but he/she was not involved in the treatment or the outcome assessment. All subjects received 4 sessions of ESWT, at once weekly intervals, at different energy levels according to their group (Fig. 1). The patient was positioned supine on the table and the affected hip joint was rotated externally while keeping every other joint in neutral position; in this position exposes, the femoral head is exposed the most. The shortest distance from the skin to the femoral head is obtained in this position and it allows concentrating more energy to the head at a lower EFD. ESWT was performed by a physician who was not involved in the selection and assessment of the patients.

\section{Outcome measures}

The primary outcome measure was pain, which was measured using a visual analog scale (VAS) [7], with 0 indicating no pain and 10 indicating maximal pain. The secondary outcome measure was 'disability', which was assessed by using the Harris hip score, Hip dysfunction and Osteoarthritis Outcome Score (HOOS), and the Western Ontario and McMaster University Osteoarthritis Index (WOMAC).

The Harris hip score is an assessment that evaluates pain, gait, activity, deformity, and range of motion; it gives a maximum of 100 points, with high score representing low symptom severity [8]. The HOOS is an assessment that evaluates daily activity (stair use, using public transportation, sitting, and managing shoes and socks) and gait (presence of a limp, support needed, and walking distance); the score gives a maximum of 100 points, with $<70$ indicating a poor result and $90-100$ indicating an excellent result [8].

The WOMAC assesses the symptoms of OA, and it is a validated disease-specific self-reporting questionnaire that refers to the 48 hours before assessment; the score ranges from 0 to 96 , with high score representing worse symptom severity [9].

We assessed the VAS, Harris hip score, HOOS, WOMAC of the subjects before and at 1,3, and 6 months after ESWT to evaluate the effectiveness of the treatment.

\section{Statistical analysis}

A student t-test was used to compare the homogeneity of baseline characteristics between the two groups (groups A and B). The treatment effect within each group

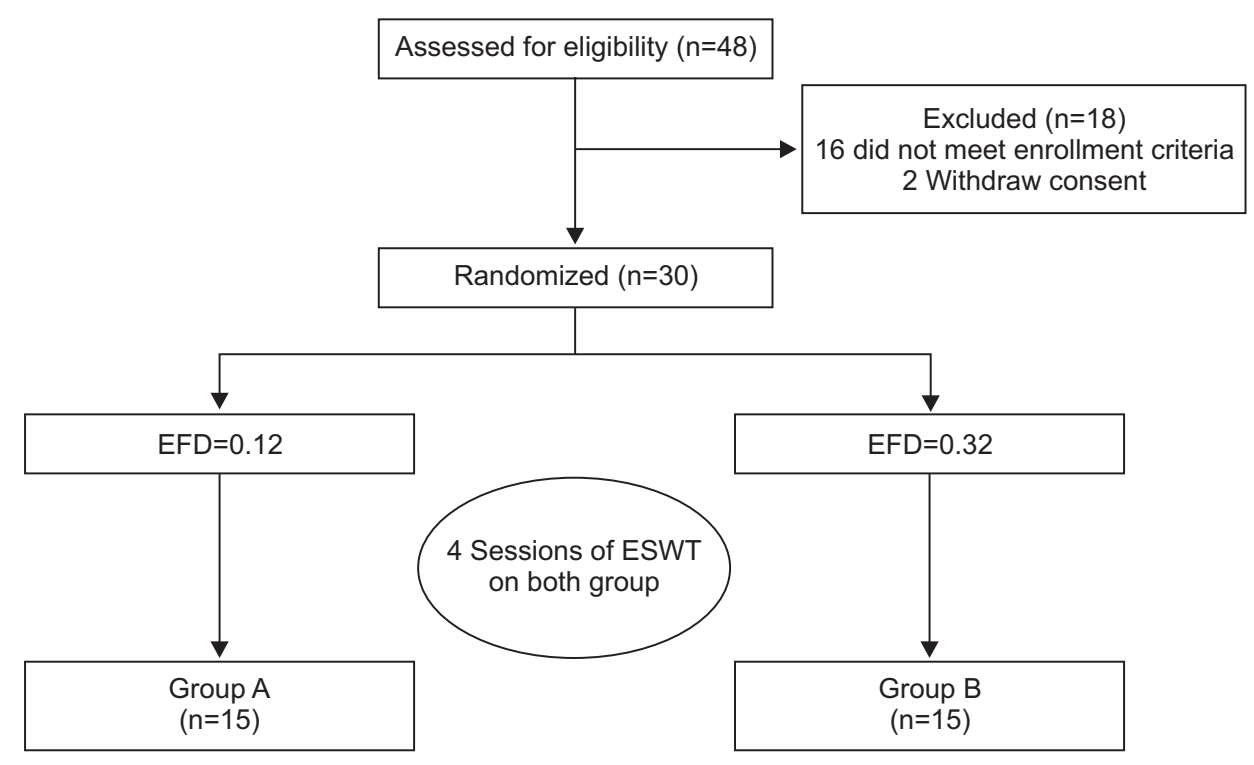

Fig. 1. A flow diagram, showing the treatment process and assessment. ESWT, extracorporeal shock wave therapy; EFD, energy flux density. 
and the difference in the treatment effect between the two groups were examined using repeated measure ANOVA. All analyses were performed with SPSS ver. 18.0 (SPSS Inc., Chicago, IL, USA). A p-value of $<0.05$ was considered statistically significant.

\section{RESULTS}

Within the group of 30 hips, 24 were male hips and 6 were female hips. The average age of patients was $64.35 \pm 5.9$ years, their average height was $167.25 \pm 7.0 \mathrm{~cm}$, their average weight was $68.9 \pm 5.8 \mathrm{~kg}$, their average body mass index (BMI) was $28.6 \pm 1.5 \mathrm{~kg} / \mathrm{m}^{2}$, and their average age at the first symptomatic onset was $49.96 \pm 9.2$ years. Their ARCO stage (I/II/III) was $3 / 6 / 6$ (group A) and $2 / 8 / 5$ (group B) (Table 1).

The average VAS, Harris hip score, HOOS, WOMAC scores were $3.28 \pm 1.81,32.42 \pm 12.28,43.22 \pm 8.90$, and $39.16 \pm 14.83$, respectively (Table 2 ). There were no significant differences in sex, age, height, weight, BMI, VAS score, Harris hip score, HOOS score, WOMAC score be- tween the, two groups prior to ESWT treatment. There was no case in which the treatment was discontinued due to the occurrence of the side effects or complications, although some patients complained of a minor discomfort like transient skin color change or swelling.

\section{Change in pain after ESWT}

At baseline, the VAS scores in groups A and B were $3.13 \pm 1.87$ and $3.43 \pm 1.74$, respectively. After 1 month, the VAS scores in the respective groups were $2.95 \pm 1.62$ and $3.12 \pm 1$.53. After 3 months, the VAS scores in the respective were $2.57 \pm 1.55$ and $2.61 \pm 1.68$, and after 6 months, the VAS scores in the respective groups were $2.04 \pm 1.47$ and $2.23 \pm 1.49$.

Compared to baseline, the VAS scores were significantly decreased over time, up to the 6-month follow-up for both groups ( $\mathrm{p}<0.05$ for time effect, $\mathrm{p}<0.05$ for grouptime interaction) (Fig. 2).

\section{Change in functional scores after ESWT}

At baseline, the Harris hip scores in groups A and B were

Table 1. The demographic data of participants

\begin{tabular}{lccc}
\hline \multicolumn{1}{c}{ Variable } & Group A (n=15) & Group B (n=15) & p-value $^{\text {a) }}$ \\
\hline Sex (male:female) & $13: 2$ & $11: 4$ & \\
Age (yr) & $64.9 \pm 6.4$ & $63.8 \pm 6.2$ & 0.286 \\
Height $(\mathrm{cm})$ & $165.8 \pm 6.9$ & $168.7 \pm 7.3$ & 0.841 \\
Weight $(\mathrm{kg})$ & $67.5 \pm 5.6$ & $70.3 \pm 5.9$ & 0.635 \\
BMI $\left(\mathrm{kg} / \mathrm{m}^{2}\right)$ & $28.8 \pm 1.7$ & $28.4 \pm 1.4$ & 0.873 \\
Age at the first symptomatic onset & $49.1 \pm 9.3$ & $50.8 \pm 8.9$ & 0.623 \\
\hline ARCO stage (I/II/III) & $3 / 6 / 6$ & $2 / 8 / 5$ & 0.715 \\
\hline
\end{tabular}

Values are presented as number or mean \pm standard deviation.

Group A, $0.12 \mathrm{~mJ} / \mathrm{mm}^{2}$; group B, $0.32 \mathrm{~mJ} / \mathrm{mm}^{2}$; BMI, body mass index; ARCO, Association Research Circulation Osseous.

${ }^{a)}$ Student t-test.

Table 2. The baseline clinical characteristics of participants

\begin{tabular}{lccc}
\hline Variable & Group A $(\mathbf{n}=\mathbf{1 5})$ & Group B $(\mathbf{n}=\mathbf{1 5})$ & p-value $^{\mathbf{a})}$ \\
\hline VAS & $3.13 \pm 1.87$ & $3.43 \pm 1.74$ & 0.678 \\
Harris hip score & $30.81 \pm 12.27$ & $34.03 \pm 12.30$ & 0.756 \\
HOOS & $44.21 \pm 8.89$ & $42.22 \pm 8.91$ & 0.812 \\
WOMAC & $39.60 \pm 14.88$ & $38.71 \pm 14.79$ & 0.793 \\
\hline
\end{tabular}

Values are presented as number or mean \pm standard deviation.

Group A, $0.12 \mathrm{~mJ} / \mathrm{mm}^{2}$; group B, $0.32 \mathrm{~mJ} / \mathrm{mm}^{2}$; VAS, visual analogue scale; HOOS, Hip dysfunction and Osteoarthritis Outcome Score; WOMAC, Western Ontario and McMaster University Osteoarthritis Index.

${ }^{a)}$ Student t-test. 


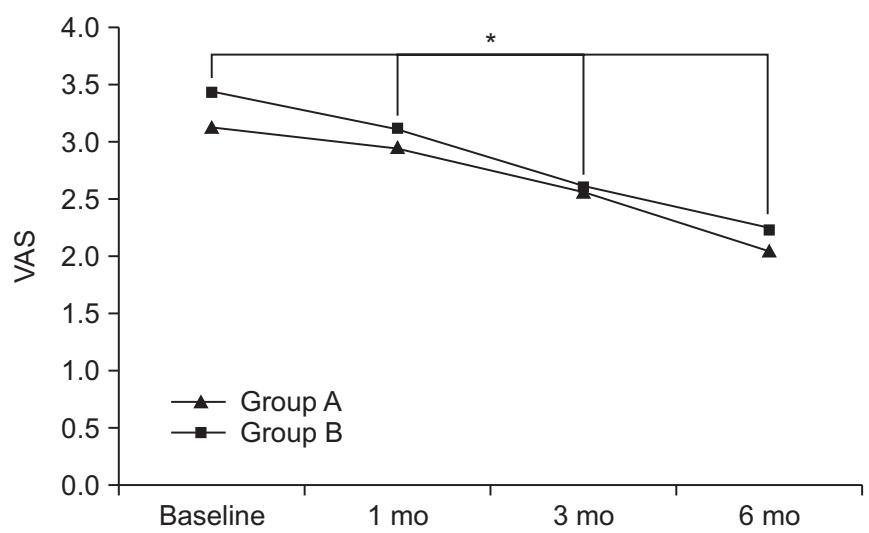

Fig. 2. The visual analogue scale (VAS) score at baseline and at 1-, 3-, and 6-month follow-ups after extracorporeal shock wave therapy in both groups. This figure shows significant improvement in the VAS score in both groups, for the entire period $\left({ }^{*} \mathrm{p}<0.05\right.$ for time effect, ${ }^{*} \mathrm{p}<0.05$ for group-time interaction). Group A, $0.12 \mathrm{~mJ} / \mathrm{mm}^{2}$ energy group; group $\mathrm{B}, 0.32 \mathrm{~mJ} / \mathrm{mm}^{2}$ energy group.

$30.81 \pm 12.27$ and $34.03 \pm 12.30$, respectively. After 1 month, the Harris hip scores in the respective groups were $37.84 \pm 12.48$ and $39.71 \pm 12.36$. After 3 months, the Harris hip scores in the respective groups were $43.64 \pm 12.75$ and $44.31 \pm 12.77$, and after 6 months, the Harris hip scores in the respective groups were $45.85 \pm 12.82$ and $46.55 \pm 12.81$.

Compared to baseline, the Harris hip scores were significantly increased over time, up to the 6-month follow up for both groups ( $\mathrm{p}<0.05$ for time effect, $\mathrm{p}<0.05$ for group-time interaction) (Fig. 3).

At baseline, the HOOSs in groups A and B were $44.21 \pm 8.89$ and $42.22 \pm 8.91$, respectively. After 1 month, the HOOSs in the respective groups were $36.05 \pm 8.72$ and $34.56 \pm 8.77$. After 3 months, the HOOSs in the respective groups were $28.36 \pm 5.31$ and $26.86 \pm 6.44$, and after 6 months, the HOOSs in the respective groups were $24.92 \pm 3.28$ and $21.88 \pm 4.91$.

Compared to baseline, the HOOSs were significant decreased over time, up to the 6-month follow up for both groups $(\mathrm{p}<0.05$ for time effect, $\mathrm{p}<0.05$ for group-time interaction) (Fig. 4).

At baseline, the WOMAC scores in groups A and B were $39.6 \pm 14.88$ and $38.71 \pm 14.79$, respectively. After 1 month, the WOMAC scores in the respective groups were $31.15 \pm 14.60$ and $32.33 \pm 14.63$. After 3 months, the WOMAC scores in the respective groups were $26.68 \pm 14.48$ and $28.78 \pm 14.53$, and after 6 months, the WOMAC scores in the respective groups were $25.02 \pm 14.31$ and $24.4 \pm 14.20$.

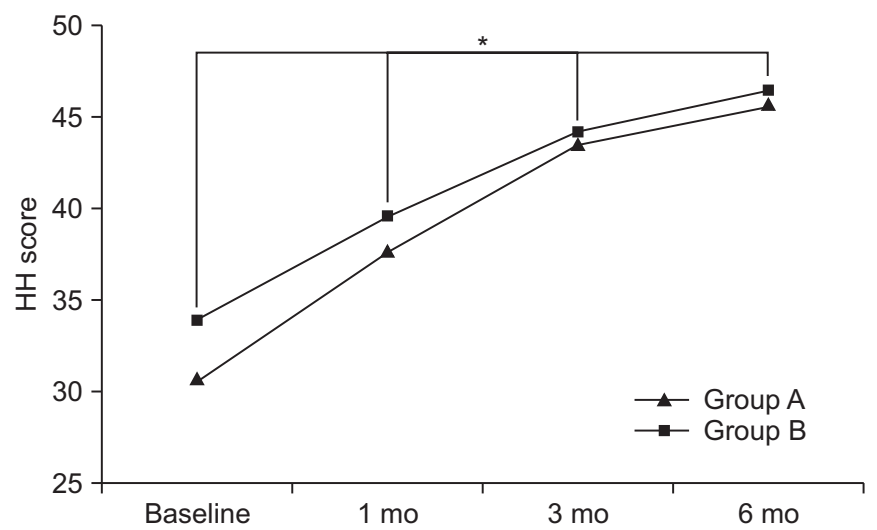

Fig. 3. The Harris hip (HH) score at baseline and at 1-, 3-, and 6-month follow-ups after extracorporeal shock wave therapy in both groups. This figure shows significant improvement in the $\mathrm{HH}$ score in both groups, for the entire period $\left({ }^{*} \mathrm{p}<0.05\right.$ for time effect, ${ }^{*} \mathrm{p}<0.05$ for group-time interaction). Group A, $0.12 \mathrm{~mJ} / \mathrm{mm}^{2}$ energy group; group $\mathrm{B}, 0.32 \mathrm{~mJ} / \mathrm{mm}^{2}$ energy group.

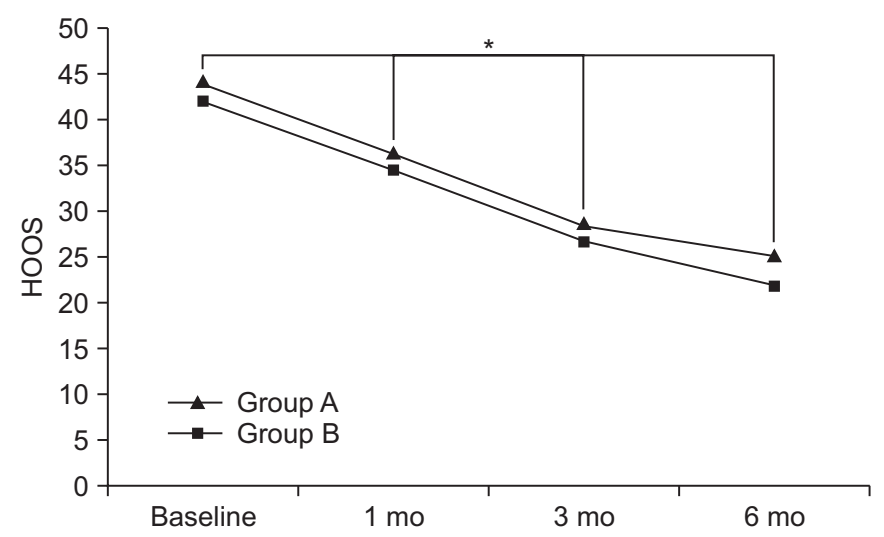

Fig. 4. The Hip dysfunction and Osteoarthritis Outcome Score (HOOS) score at baseline and at 1-, 3-, and 6 -month follow-ups after extracorporeal shock wave therapy in both groups. This figure shows significant improvement in the HOOS score in both groups, for entire period $\left({ }^{*} p<0.05\right.$ for time effect, ${ }^{*} \mathrm{p}<0.05$ for group-time interaction). Group A, $0.12 \mathrm{~mJ} / \mathrm{mm}^{2}$ energy group; group $\mathrm{B}, 0.32 \mathrm{~mJ} / \mathrm{mm}^{2}$ energy group.

Compared to baseline, the WOMAC scores were significantly decreased over time, up to the 6-month follow-up for both groups ( $\mathrm{p}<0.05$ for time effect, $\mathrm{p}<0.05$ for grouptime interaction) (Fig. 5).

\section{DISCUSSION}

There are many ongoing studies on non-surgical treat- 


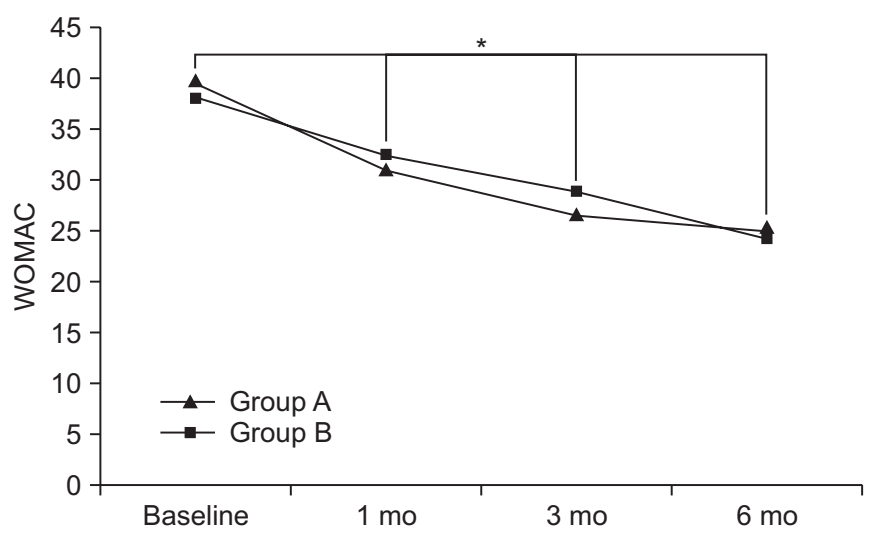

Fig. 5. The Western Ontario and McMaster University Osteoarthritis Index (WOMAC) score at baseline and at 1-, 3-, and 6-month follow-ups after extracorporeal shock wave therapy in both groups. This figure shows significant improvement in the WOMAC score in both groups, for entire period $\left({ }^{*} \mathrm{p}<0.05\right.$ for time effect, ${ }^{*} \mathrm{p}<0.05$ for group-time interaction). Group A, $0.12 \mathrm{~mJ} / \mathrm{mm}^{2}$ energy group; group $\mathrm{B}, 0.32 \mathrm{~mJ} / \mathrm{mm}^{2}$ energy group.

ments of AVN of the femoral head, especially ESWT; the effects on alleviation of symptoms and improvement of function are undergoing the verification process. Previous studies reported that ESWT is an effective treatment for AVN of the femoral head. Chen et al. [10] included 17 patients with bilateral hip osteonecrosis who were treated with ESWT ( $28 \mathrm{kV}, 6,000$ impulses) on one hip and hip arthroplasty on the other hip, and both procedures resulted in favorable pain and functional scores. In 2005, Wang et al. [11] compared 23 patients with 29 hips treated with ESWT ( $28 \mathrm{kV}, 6,000$ impulses) and 25 patients with 28 hips treated by core decompression, and ESWT showed more effectiveness than core decompression in the short term. Ludwig et al. [12] reported comparable clinical results of ESWT in the treatment of patients with early stages (1-3) of osteonecrosis of the femoral head (ONFH), and the visual analog pain scores were decreased from 8.5 to 1.2 at 1 year after ESWT treatment while the Harris hip scores were increased from 43.3 to 92 . There are other numerous data indicating the therapeutic effectiveness of ESWT $[3-6,13,14]$. Our research on the low-energy flux density ESWT treatment up to the 6-month follow-up showed that it has a significant effect in treating the symptoms of AVN of the femoral head; both treatment methods that we used showed their therapeutic effectiveness; however, there was no significant difference between the two groups regardless of the difference in EFD per shock levels.

The mechanism of ESWT is not completely understood; however, it is assumed that ESWT affects the disease process at the molecular level; ESWT shockwave treatment significantly up-regulated cell proliferation, vascular endothelial growth factor (VEGF), alkaline phosphatase, bone morphogenetic protein-2 (BMP2), runt-related transcription factor 2 (RUNX2), and osteocalcin mRNA expressions and resulted in more mature mineralized nodules [1]. Furthermore, ESWT was suggested to promote angiogenesis and bone remodeling and to exert a regenerative effect through the induction of the NO pathway in AVN of the femoral head $[13,15]$.

ESWT is a new therapeutic technology and it has the potential to replace surgery in patients with AVN of the femoral head without any surgical risks. In this research, ESWT at low-EFD seems to be beneficial in-patients with the early stage of AVN of the femoral head.

This study has several limitations. Because the control group that follows the ISMST ESWT protocol was not employed, we could not make a statistical comparison between our results and the ISMST recommendations. Due to the lack of the control group, comparisons with other conservative treatments of AVN of the femoral head could not be made. Also, our research did not include the evaluations of the osteogenic growth factors and the hematologic tests, which were reported to be the influential factors in the ESWT treatment; thus, the study of the treatment effects at the anatomical, molecular, and biochemical levels remains incomplete. We suggest that further studies including the control group and other evaluations of various factors, such as a larger patient pool, sex, age, AVN stage, operative technique and so on, should be performed.

Although our study lacks a complete laboratory testing of the effectiveness of the treatment, we conclude that the study has its own significance because the patients experienced alleviation of pain and improvement of joint function after the treatment. Therefore, ESWT at lowEFD, which can be readily adopted in the general clinical environment, has a significant importance. Researches on ESWT for treating AVN of the femoral head have not been actively performed due to the technical limitation. However, with application of this experimental lowenergy density ESWT, we expect that numerous attempts 
will be made to treat AVN with greater ease; thus, helping to reduce patient discomfort.

\section{CONFLICT OF INTEREST}

No potential conflict of interest relevant to this article was reported.

\section{REFERENCES}

1. Wang CJ, Cheng JH, Huang CC, Yip HK, Russo S. Extracorporeal shockwave therapy for avascular necrosis of femoral head. Int J Surg 2015;24(Pt B):184-7.

2. Assouline-Dayan Y, Chang C, Greenspan A, Shoenfeld $\mathrm{Y}$, Gershwin ME. Pathogenesis and natural history of osteonecrosis. Semin Arthritis Rheum 2002;32:94-124.

3. Mont MA, Carbone JJ, Fairbank AC. Core decompression versus nonoperative management for osteonecrosis of the hip. Clin Orthop Relat Res 1996;324:16978.

4. Wong T, Wang CJ, Hsu SL, Chou WY, Lin PC, Huang CC. Cocktail therapy for hip necrosis in SARS patients. Chang Gung Med J 2008;31:546-53.

5. Revell P. Book review: Bone circulation and vascularization in normal and pathological conditions. J Anat 1994;184(Pt 2):429.

6. Kusz D, Franek A, Wilk R, Dolibog P, Blaszczak E, Wojciechowski $\mathrm{P}$, et al. The effects of treatment the avascular necrosis of the femoral head with extracorporeal focused shockwave therapy. Ortop Traumatol Rehabil 2012;14:435-42.

7. Carlsson AM. Assessment of chronic pain. I. Aspects of the reliability and validity of the visual analogue scale. Pain 1983;16:87-101.

8. Harris WH. Traumatic arthritis of the hip after dislo- cation and acetabular fractures: treatment by mold arthroplasty. An end-result study using a new method of result evaluation. J Bone Joint Surg Am 1969;51:73755.

9. Bellamy N, Campbell J, Stevens J, Pilch L, Stewart C, Mahmood Z. Validation study of a computerized version of the Western Ontario and McMaster Universities VA3.0 Osteoarthritis Index. J Rheumatol 1997;24:2413-5.

10. Chen JM, Hsu SL, Wong T, Chou WY, Wang CJ, Wang FS. Functional outcomes of bilateral hip necrosis: total hip arthroplasty versus extracorporeal shockwave. Arch Orthop Trauma Surg 2009;129:837-41.

11. Wang CJ, Wang FS, Huang CC, Yang KD, Weng LH, Huang HY. Treatment for osteonecrosis of the femoral head: comparison of extracorporeal shock waves with core decompression and bone-grafting. J Bone Joint Surg Am 2005;87:2380-7.

12. Ludwig J, Lauber S, Lauber HJ, Dreisilker U, Raedel R, Hotzinger H. High-energy shock wave treatment of femoral head necrosis in adults. Clin Orthop Relat Res 2001;387:119-26.

13. Wang CJ, Wang FS, Ko JY, Huang HY, Chen CJ, Sun YC, et al. Extracorporeal shockwave therapy shows regeneration in hip necrosis. Rheumatology (Oxford) 2008;47:542-6.

14. Wang CJ, Huang CC, Wang JW, Wong T, Yang YJ. Longterm results of extracorporeal shockwave therapy and core decompression in osteonecrosis of the femoral head with eight- to nine-year follow-up. Biomed J 2012;35:481-5.

15. Yin TC, Wang CJ, Yang KD, Wang FS, Sun YC. Shockwaves enhance the osteogenetic gene expression in marrow stromal cells from hips with osteonecrosis. Chang Gung Med J 2011;34:367-74. 